\title{
Donafenib as a first-line monotherapy for advanced hepatocellular carcinoma
}

\author{
Bo-Han Zhang", Yun-Shi Cai", Li Jiang, Jia-Yin Yang \\ Department of Liver Surgery, Liver Transplantation Center, West China Hospital of Sichuan University, Chengdu, China \\ \#These authors contributed equally to this work. \\ Correspondence to: Prof. Jia-Yin Yang; Prof. Li Jiang. Department of Liver Surgery, Liver Transplantation Center, West China Hospital of Sichuan \\ University, 37 Guoxue Lane, Wuhou District, Chengdu 610041, China. Email: docjackyang@163.com; j1339@126.com.
}

Submitted Jul 23, 2021. Accepted for publication Sep 09, 2021.

doi: 10.21037/hbsn-21-304

View this article at: https://dx.doi.org/10.21037/hbsn-21-304

We are optimistic about Professor Shukui Qin and colleagues' electrifying results regarding the efficacy and safety of donafenib (a modified form of sorafenib) over sorafenib in treating advanced hepatocellular carcinoma (aHCC) (1). Following the landmark sorafenib trial in 2008 (2), the present ZGDH3 trial is the first and only first-line head-to-head phase 2-3 trial to demonstrate that monotherapy is superior to sorafenib in terms of overall survival (OS) for aHCC. For the ZGDH3 trial, Qin et al. randomly assigned 668 eligible patients to receive $200 \mathrm{mg}$ of donafenib or $400 \mathrm{mg}$ of sorafenib, orally, twice daily, with open-label administration [donafenib ( $n=334)$, sorafenib $(n=334)$ ]. The primary endpoint was OS and secondary endpoints included progression-free survival (PFS), time to progression, objective response rate (ORR), disease control rate (DCR), survival rates at 6, 9, 12, and 18 months, and safety.

In the full analysis set [FAS, donafenib $(n=328)$ and sorafenib ( $n=331)$ ], donafenib showed not only noninferiority, but also superiority, over sorafenib for median OS [12.1 vs. 10.3 months; hazard ratio (HR), 0.831; $95 \%$ confidence interval (CI), 0.699 to $0.988 ; \mathrm{P}=0.0245]$. This was because the upper limit of the $95 \%$ CI for HR was $<1.08$ (for non-inferiority) and $<1.00$ (for superiority) (1). The donafenib arm had less drug-related grade $\geq 3$ adverse events than the sorafenib arm $[125$ (38\%) vs. 165 (50\%); $\mathrm{P}=0.0018]$. Compared with patients receiving sorafenib, the advantages of PFS (3.7 vs. 3.6 months; $\mathrm{P}=0.0570$ ), ORR ( $4.6 \%$ vs. $2.7 \% ; \mathrm{P}=0.2448)$ and $\mathrm{DCR}(30.8 \%$ vs. $28.7 \% ; \mathrm{P}=0.5532)$ in patients receiving donafenib were slim and statistically insignificant. These data provide some conclusions, while also raising several concerns.
Even though OS improved in the donafenib arm, the PFS, ORR and DCR were akin to those in the sorafenib arm. With their modest clinical benefits, issues regarding the strict control of cost-effectiveness and selection of potential specific populations who may respond to donafenib treatment merit further inquiry. For instance, combined use of atezolizumab (anti-PD-L1 antibody) and bevacizumab (anti-VEGF antibody) ("T+A") improved OS and PFS compared with sorafenib alone among aHCC patients without prior systematic therapy (3). This treatment has also been endorsed as the standard of care in first-line aHCC therapy (4). However, Zhang et al. have concluded that " $\mathrm{T}+\mathrm{A}$ " is not cost-effective, with an ICER (incremental cost-effectiveness ratio) of \$322,500 per QALY (quality-adjusted life year) compared with sorafenib (5). Furthermore, to optimize donafenib's therapeutic efficacy, we have to hunt for and validate biomarkers to screen individuals who would be likely to respond. Despite gloomy results from biomarker-driven targeted therapy, the highthroughput sequencing, and the precision medicine that it underpins, continue to push forward the implementation of novel clinical trials (6).

Researchers have acknowledged quality of life (QoL) as an indispensable indicator for decision-making in the battle against HCC, especially over the long term $(7,8)$. We think that evaluating patients' QoL would provide more insight into donafenib since a long-term follow-up may be a leading contributor to the OS improvement donafenib caused. The Kaplan-Meier curve in figure 2A supports this conclusion. The trajectories of the two curves continue to intertwine until they diverge at about 15 months, and the degree 
of deviation (i.e., the distance between the two curves) does not change with time. Moreover, as indicated in the stratified analysis, the difference in survival rates between the two groups was statistically significant at neither 6,9 , nor 12 months, and it was not until 18 months that the donafenib arm showed a statistically superior survival rate. Moreover, we obtained the follow-up time data for the previous phase 3 trials regarding first-line aHCC therapies by extracting it from the original context and computing the survival data using the reverse Kaplan-Meier method via the 'IPDfromKM' package (9). We found that the ZGDH3 trial had a long follow-up (Table 1).
In addition, the integration of this improved systemic treatment modality into a broad arsenal of locoregional therapeutic techniques such as transarterial chemoembolization may demonstrate synergistic effects that inhibit both angiogenic factors and tumor growth (10). Finally, given that most of the patients enrolled in FAS (97.4\%) were Child-Pugh class A, an investigation of targeted strategies in patients with aHCC who have less compensated liver function is warranted. This is because in clinical practice, most HCC patients are already at an advanced stage when diagnosed, with an elevated probability of liver abnormality.

Table 1 Phase III trials of first-line therapies for advanced hepatocellular carcinoma

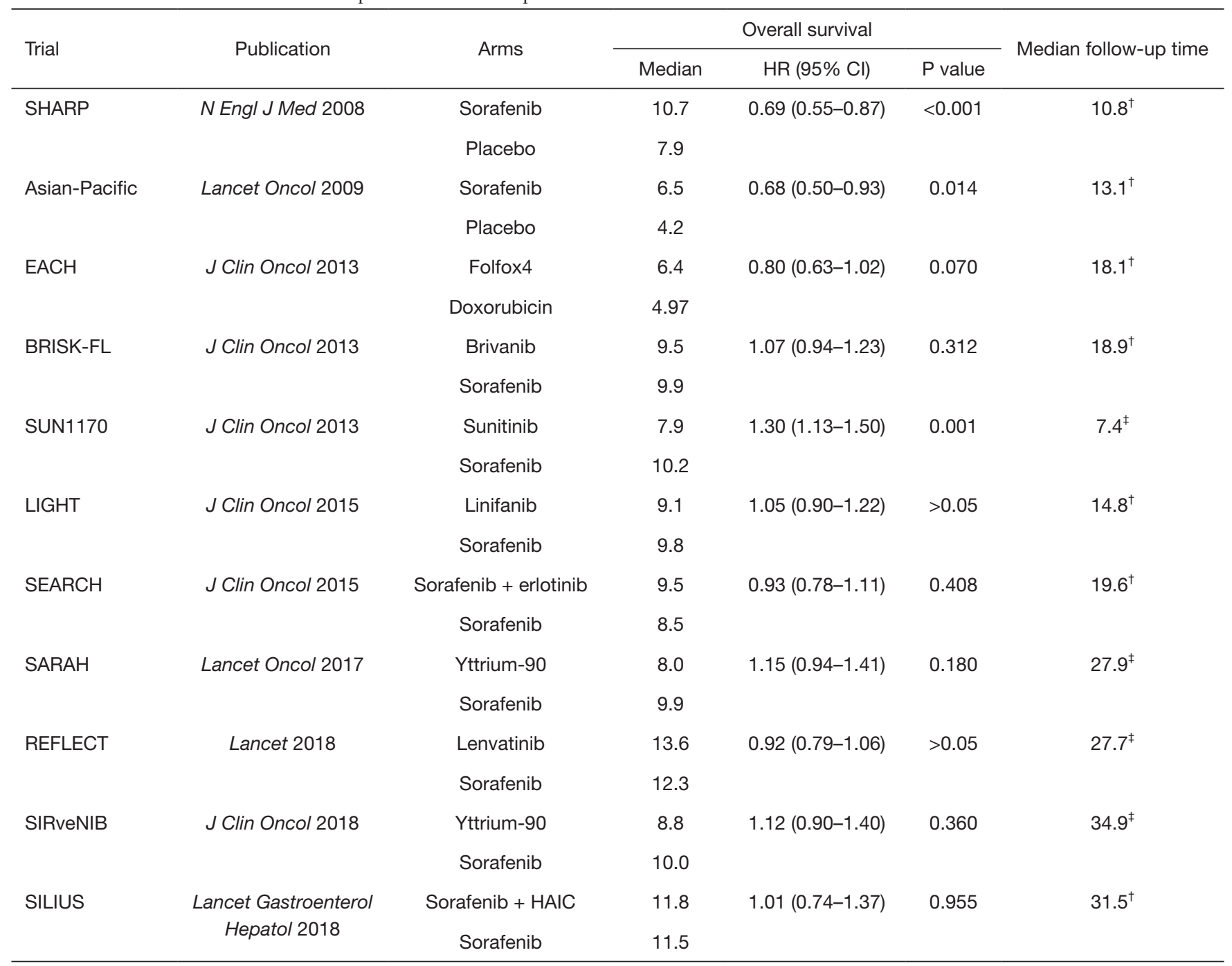

Table 1 (continued) 
Table 1 (continued)

\begin{tabular}{|c|c|c|c|c|c|c|}
\hline Trial & Publication & Arms & \multicolumn{3}{|c|}{ Overall survival } & Median follow-up time \\
\hline \multirow[t]{2}{*}{ CheckMate 459} & Ann Oncol 2019 & Nivolumab & 16.4 & $0.85(0.72-1.02)$ & 0.075 & $29.7^{\dagger}$ \\
\hline & & Sorafenib & 14.7 & & & \\
\hline CALGB 80802 & JAMA Oncol 2019 & Sorafenib + doxorubicin & 9.3 & $1.05(0.83-1.31)$ & 0.680 & $36.1^{\ddagger}$ \\
\hline \multirow[t]{2}{*}{ SORAMIC } & J Hepatol 2019 & Sorafenib + SIRT & 12.1 & $1.01(0.81-1.25)$ & 0.953 & $9.4^{\ddagger}$ \\
\hline & & Sorafenib & 11.4 & & & \\
\hline IMbrave150 & N Engl J Med 2020 & $\begin{array}{c}\text { Atezolizumab + Bevaci- } \\
\text { zumab }\end{array}$ & NE & $0.58(0.42-0.79)$ & $<0.001$ & $8.6^{\ddagger}$ \\
\hline
\end{tabular}

${ }^{\dagger}$, obtained by calculating survival data using the reverse Kaplan-Meier method; ${ }^{\ddagger}$, obtained from the source text. HAIC, hepatic arterial infusion chemotherapy; SIRT, selective internal radiation therapy; NE, not evaluable; HR, hazard ratio; Cl, confidence interval.

\section{Acknowledgments}

Funding: This work was funded by the National Natural Science Foundation of China (No. 81770653 and 82070674), the Clinical New Technology Project of West China Hospital, Sichuan University (No. 20HXJS012) and the Sichuan Science and Technology Program (2019YFS0204).

\section{Footnote}

Provenance and Peer Review: This article was a standard submission to the Hepatobiliary Surgery and Nutrition. The article did not undergo external peer review.

Conflicts of Interest: All authors have completed the ICMJE uniform disclosure form (available at https://hbsn. amegroups.com/article/view/10.21037/hbsn-21-304/coif). The authors have no conflicts of interest to declare.

Ethical Statement: The authors are accountable for all aspects of the work in ensuring that questions related to the accuracy or integrity of any part of the work are appropriately investigated and resolved.

Open Access Statement: This is an Open Access article distributed in accordance with the Creative Commons
Attribution-NonCommercial-NoDerivs 4.0 International License (CC BY-NC-ND 4.0), which permits the noncommercial replication and distribution of the article with the strict proviso that no changes or edits are made and the original work is properly cited (including links to both the formal publication through the relevant DOI and the license). See: https://creativecommons.org/licenses/by-nc-nd/4.0/.

\section{References}

1. Qin S, Bi F, Gu S, et al. Donafenib Versus Sorafenib in First-Line Treatment of Unresectable or Metastatic Hepatocellular Carcinoma: A Randomized, Open-Label, Parallel-Controlled Phase II-III Trial. J Clin Oncol 2021;39:3002-11.

2. Llovet JM, Ricci S, Mazzaferro V, et al. Sorafenib in advanced hepatocellular carcinoma. $\mathrm{N}$ Engl J Med 2008;359:378-90.

3. Finn RS, Qin S, Ikeda M, et al. Atezolizumab plus Bevacizumab in Unresectable Hepatocellular Carcinoma. N Engl J Med 2020;382:1894-905.

4. Llovet JM, Kelley RK, Villanueva A, et al. Hepatocellular carcinoma. Nat Rev Dis Primers 2021;7:6.

5. Zhang X, Wang J, Shi J, et al. Cost-effectiveness of Atezolizumab Plus Bevacizumab vs Sorafenib for Patients With Unresectable or Metastatic Hepatocellular 
Carcinoma. JAMA Netw Open 2021;4:e214846.

6. Huang A, Yang XR, Chung WY, et al. Targeted therapy for hepatocellular carcinoma. Signal Transduct Target Ther 2020;5:146.

7. Zhou K, Fountzilas C. Outcomes and Quality of Life of Systemic Therapy in Advanced Hepatocellular Carcinoma. Cancers (Basel) 2019;11:861.

8. Li D, Sedano S, Allen R, et al. Current Treatment Landscape for Advanced Hepatocellular Carcinoma:
Patient Outcomes and the Impact on Quality of Life. Cancers (Basel) 2019;11:841.

9. Liu N, Zhou Y, Lee JJ. IPDfromKM: reconstruct individual patient data from published Kaplan-Meier survival curves. BMC Med Res Methodol 2021;21:111.

10. Kim HY, Park JW. Clinical trials of combined molecular targeted therapy and locoregional therapy in hepatocellular carcinoma: past, present, and future. Liver Cancer 2014;3:9-17.

Cite this article as: Zhang BH, Cai YS, Jiang L, Yang JY. Donafenib as a first-line monotherapy for advanced hepatocellular carcinoma. HepatoBiliary Surg Nutr 2021;10(5):737-740. doi: 10.21037/hbsn-21-304 